\title{
Integrating Optical Imaging of Mummy Mask Cartonnage
}

\author{
M.B. Toth, University College London (UCL) UK, R.B. Toth Associates, Oakton VA, USA; C.A. Toth, Duke University, Durham NC, \\ USA; W. Christens-Barry, UCL, London UK, Equipoise Imaging, Ellicott City MD, USA; S. Farsiu, Duke University, Durham NC, USA; \\ G. Li, Duke University, Durham NC, USA; A. Gibson, UCL, London UK; M. Terras, UCL, London UK
}

\begin{abstract}
This rapid development and testing project captured data from multiple digital imaging techniques to try to see texts in papyrus mâché mummy mask cartonnage layers. Prior studies by other scholars destroyed the masks to access the papyri, denying future researcher access to the primary historical artefacts. This international, multidisciplinary project assessed the feasibility of integrating non-destructive digital imaging technologies to make texts visible in images of papyrus in mummy mask cartonnages for open research and analysis. The team used both optical multispectral imaging and optical coherence tomography at Duke University to try to detect the presence of text and offer scientifically valid approaches for documenting the initial state of objects and their production for future research and analysis without their destruction.
\end{abstract}

\section{Background}

The awareness that ancient Egyptian objects could be filled with manuscripts has led papyrologists to destroy cartonnage, mummy masks and other material to retrieve their contents. Specialists' recognition of the problems connected with such practice has increased, and new, less invasive techniques have been developed to avoid the destruction of important historical evidence. The decision to eventually dismount cartonnage involves careful evaluations of the pros and cons and of the methods to be followed. Yet dealers and collectors have continued the practice of dissolving cartonnage to retrieve papyri, especially in the "golden age of collection" during the early 20th Century, but continuing to this day.[1] Some collectors capitalize on opportunities to multiply their earnings or focus on manuscripts, without recognizing the issues involved with the destruction of ancient artefacts.

Cultural heritage objects are currently caught up in the destruction and commercial trade in artifacts to fund terrorist groups, including the Islamic State and Taliban. Combined with the inability of some nations to devote resources to the enforcement of cultural heritage laws - as is the case in Egypt since the revolutions - increasing numbers of artifacts are entering the market illicitly. In these cases, the damage produced to cultural heritage is even greater since little if any attention to the methods employed and to the recording of the state of the original object and the process of dismantling it to destruction.

The application of advanced imaging techniques has the potential to improve studies of papyri encapsulated in ancient artefacts and will potentially solve the problem of invasive, destructive approaches to the remains of our ancient past. In recognition of this, Arcadia provided seed funding for this exploratory, pilot project by international partners and collections. Between November 2015 and December 2016, they tested the feasibility of non-destructive imaging of multiple layers of papyrus found in Egyptian mummy cartonnages. This project has made all its data freely available, and published lessons-learned on findings and imaging methodologies for further research, including this paper on the optical image methods only.

\section{Goals and Methods}

Imaging of Egyptian Ptolemaic mummy mask cartonnage requires penetration of the paint and papyrus layers, as well as binders and other materials from which the artefact was constructed. A multidisciplinary team conducted this nondestructive imaging of mummy cartonnage and surrogates in an integrated effort to try to offer methods to avoid the dismounting and destruction of the ancient artefacts. This built on the extensive experience of teams from multiple institutions that pioneered planning, management and imaging with advanced imaging systems. The project team implemented a phased and agile approach to develop and apply increasingly complex imaging, processing and integration techniques.

The goals of this project were aggressive and pushed the limits of existing technology and the laws of physics:

"This project includes the following goals, with increasing levels of difficulty:

1. Provide images that reveal existence of text on papyrus beneath the surface colorants.

2. Provide images that reveal texts on various layers of papyrus in the cartonnage.

3. Process images to separate the layers of text for assessment of the textual content.

4. Process images to differentiate text layers with sufficient legibility for transcription."

The initial project proposal noted: "If unsuccessful in meeting these goals for technical reasons (especially the latter three goals), this project will have at least documented the strengths and weaknesses of various scientific methods to allow others to build on this non-destructive approach for further study and documentation of mummy masks and other objects."

The overall imaging program comprised multiple imaging technologies integrated into a process flow that started with assessment of the objects' fragility and composition. This was followed by baseline multispectral imaging of all objects (Fig. 1).

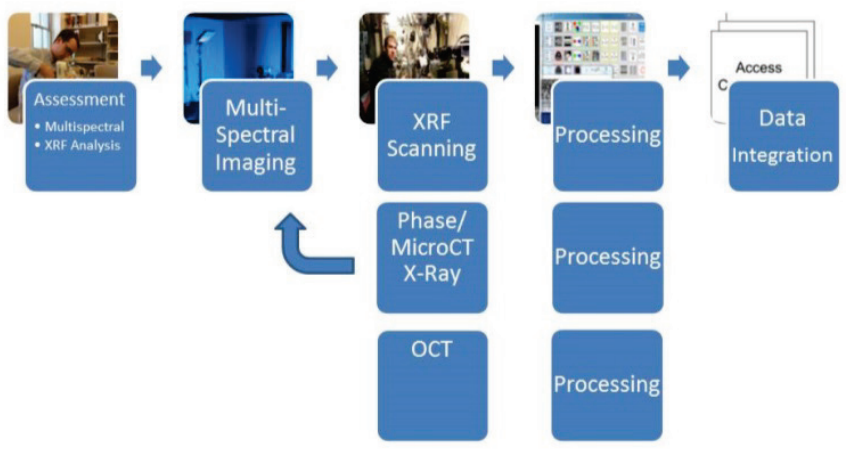

Figure 1. Mummy cartonnage imaging integrated process flow 
This baseline multispectral imaging was followed by Optical Coherence Tomography (OCT) at Duke University and imaging with the higher energy levels of $\mathrm{x}$-rays, as well as terahertz imaging (which is not addressed in this paper). All images were then converted to TIFFs and JPEGs and processed to offer better visualization of the key areas of interest. The project team also documented opportunities for standardizing data output while implementing data and program management techniques for the large amounts of data to be captured.[2] The team imaged actual cartonnage (Fig. 2), as well as a small fragment and surrogate test objects that did not require conservation care, handling and transport. It addressed the goal of not only to collecting and processing images with available digital imaging technologies, but cross-disciplinary collaboration in use of these images for advanced processing and analysis.

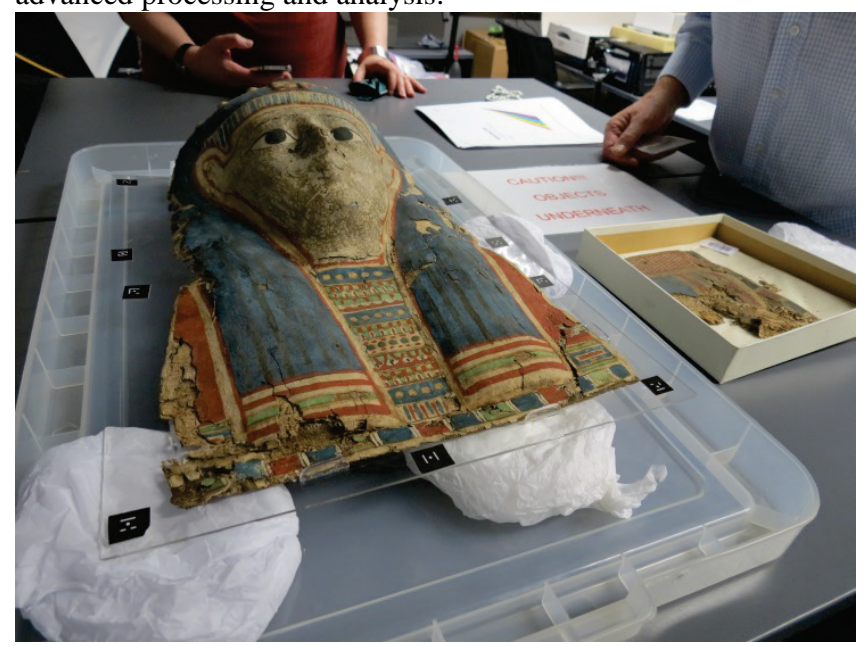

Figure 2. Petrie Museum cartonnage mummy mask and cartonnage fragment

\section{Optical Imaging of Mummy Cartonnage}

Optical imaging, especially multispectral, served as baseline imaging for the project, as it offers potential to characterize the mummy cartonnage inks and pigments on the papyri surface layers, and some under layers, depending on the thickness of the papyrus and obscuring pigments. The greatest potential to detect under-text is in the longer infrared wavelengths.

Both multispectral and OCT imaging were used to detect texts optically. Multispectral imaging offered good spatial area and spectral resolution in multiple narrowband wavelengths, and therefore offered an overall assessment and analysis of papyrus cartonnage. OCT was used to detect and assess various layers of papyrus and ink in smaller spatial areas in the infrared wavelengths. It has been used effectively for a range of medical and non-medical applications to detect cell layers and painting layers.[3]

At Duke University, Egyptian mummy cartonnage P.Duk.inv 716 and P.Duk.inv 588 (b) from the Duke University Library and the standard samples were imaged using the portable multispectral imaging system and Duke OCT systems.

\section{Multispectral Imaging}

Multispectral imaging served as a baseline imaging technique for all objects included in this project. This used an integrated system that includes commercial-off-the-shelf hardware and software to capture high spectral and spatial resolution image sets.
The multispectral imaging system used on this project was similar at all sites, comprising a Phase One IQ260 Achromatic camera with a 16-bit monochrome sensor and $120 \mathrm{~mm}$ macro lens captured 60 megapixel (MP) images (8964 x 6716), with current larger sensors now capturing 100 MP images. Because monochrome sensors do not employ a Bayer color filter array, each pixel is able to capture all of the light at wavelength within its sensitivity range without attenuation or color effects. Avoidance of individual pixel filters of a Bayer array offers many advantages over use of a color sensor, including avoidance of resolution loss that accompanies color interpolation in color sensors and the ability to optimize camera settings for captures at each individual illumination wavelength.

Reflectance images were captured using 12 illumination wavelengths from the ultraviolet (UV) through the visible and into the infrared (IR) with narrowband illumination from low heat and low maintenance, long-lifetime light emitting diodes (LED). A 6position motorized filter wheel containing 2 "' x 2" square optical glass filters, increases the range of captured information to include fluorescence emissions from the object.

The object and camera are not moved during imaging so that all the images are registered with each other. The camera was mounted at nadir above the object on a fixed copy stand, with the filter wheel below. The LED light panels were placed approximately 30-45 inches to the right and to the left of the camera lens axis, which was oriented in a vertical, downwardlooking configuration (Fig 3). The incidence angle of the illumination panels was $30^{\circ}-45^{\circ}$ from the horizontal.

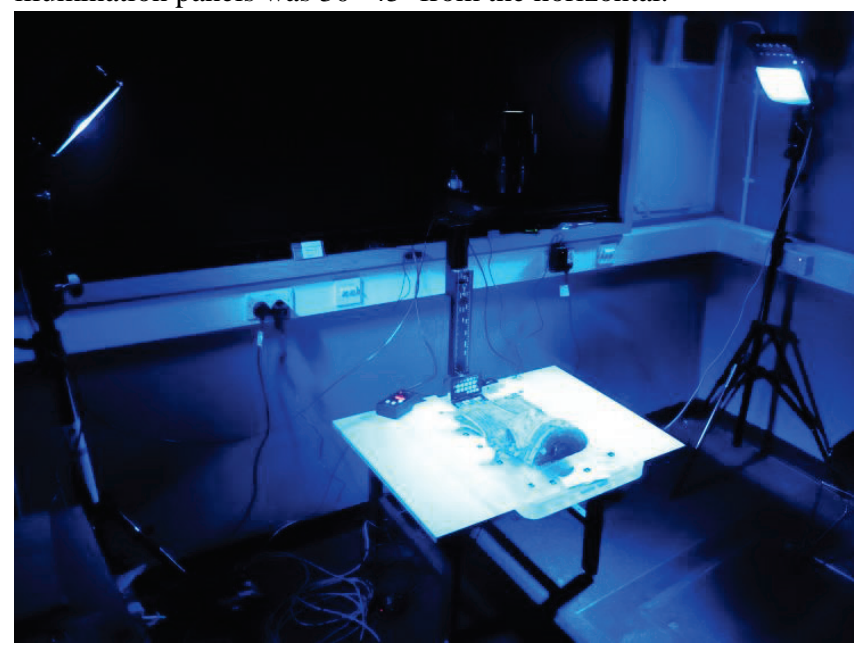

Figure 3. Multispectral Imaging system setup for UCL mummy mask imaging

Two sequences were captured of each cartonnage fragment: one of the recto side and one of the verso. For each sequence, 17 images were acquired: 12 reflectance images and 5 fluorescence images. The fluorescence images were captured with the fragment illuminated in the ultraviolet $(370 \mathrm{~nm})$ and a deep blue $(448 \mathrm{~nm})$ light, and violet, green and red long-pass filters to only allow the desired wavelengths of light to pass through the lens.

A key precept of multispectral imaging is image processing that employs sequences of images - not just single images - to provide better visualization and insight into the spectral response of the object. Combinations of wavelengths identified using numerical techniques like principal components analysis often 
correlate with the different classes of content, such as papyrus, inks, different color pigments, gesso and contaminants. Processing the captured images proved valuable in identifying which characters of a text were drawn with one type of ink and distinguishing these from paints, contaminants and different inks.

Multispectral imaging and image processing allowed users to discern spectral differences too faint to be discerned visually or in an image from a single wavelength - allowing visual recognition of faint content that would otherwise be undetected. This includes text under very thin layers of gesso. While the thin layer of gesso obscured text in Duke papyrus cartonnage fragment P Duke Inv 716 in visible wavelengths (Fig. 4), processing of images with UV and IR wavelengths revealed important text (Fig.5). This prompted Duke researchers to note "This is pretty awesome."[4]

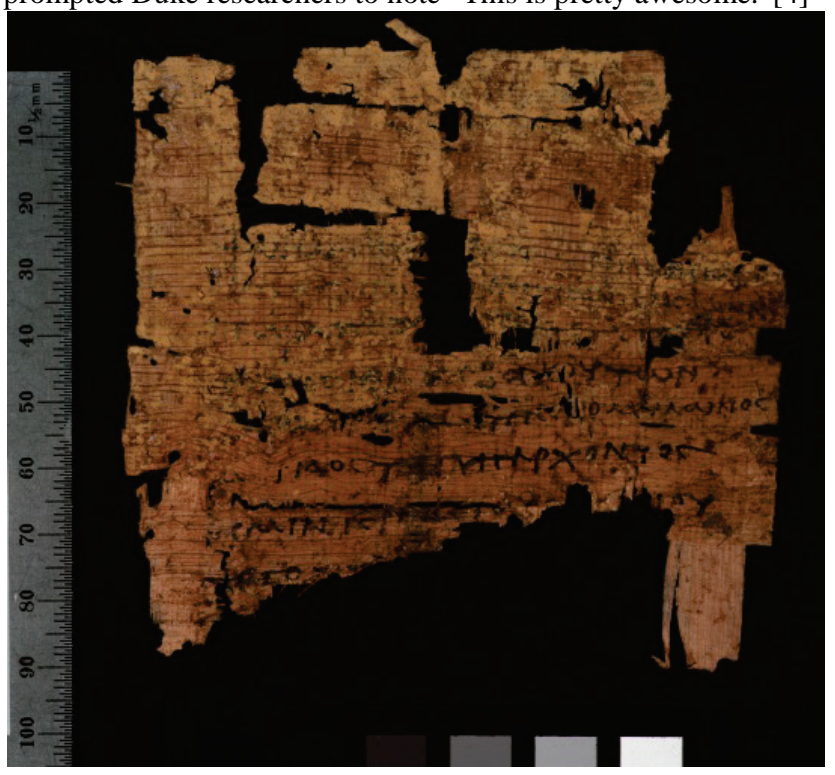

Figure 4. Image of Duke papyrus cartonnage with gesso obscuring text

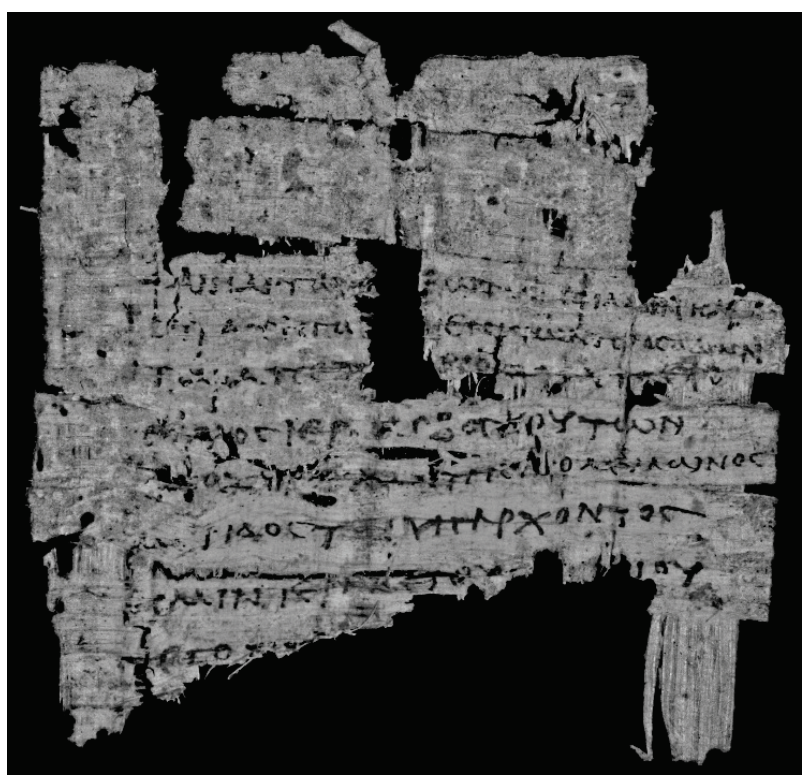

Figure 5. Processed image of Fig 4 Duke papyrus cartonnage revealing text

\section{Optical Coherence Tomography}

Personnel at the Duke University Eye Center and Biomedical Engineering Department conducted Spectral Domain OCT (SDOCT) imaging of papyri samples from the Duke Library and the surrogate papyrus samples. In SD-OCT imaging, coherent light is projected on the object, which then scatters and reflects off opaque layers to create an image. In the Duke Eye Center, SD-OCT imaging is used in the evaluation and surgical treatment of vitreoretinal diseases in infants, children and adults, with clinical application of OCT imaging in surgery and at the bedside.

Two different SD-OCT Systems were used: an Envisu R2200 Ultra-high-resolution system at $800 \mathrm{~nm}$ and a Wasatch Photonics Spark DRC system at 740-920 nm (Fig 6). The objects imaged included papyrus mâché mummy fragments with painted surfaces from the Duke Library.

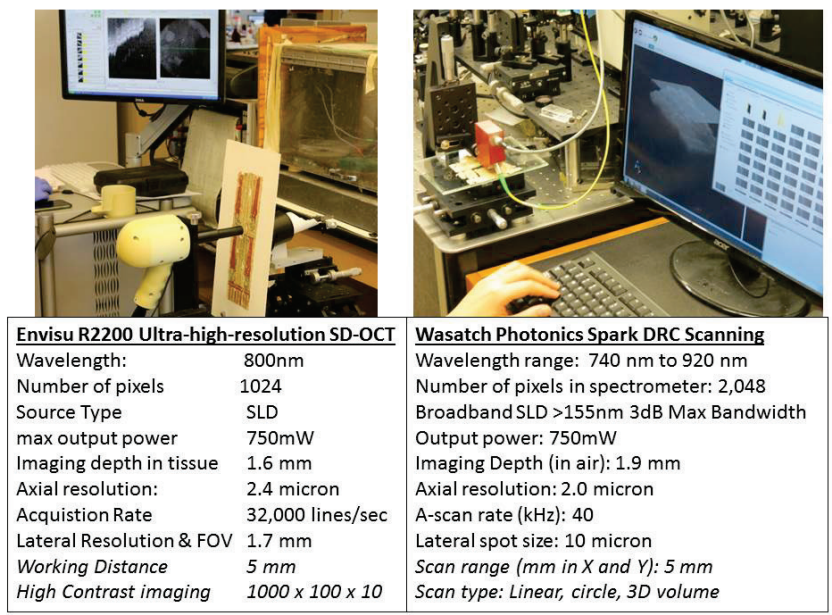

Figure 6. SD-OCT systems with mummy cartonnage at Duke University

The Envisu system is a handheld device used for ophthalmological research to image the fundus of the eye through the lens to reveal medical issues. This yields a small circular field of view of about $1.7 \mathrm{~mm}$, as well as an interferometric depth scan of about $1.6 \mathrm{~mm}$ of the same area (Fig. 7).

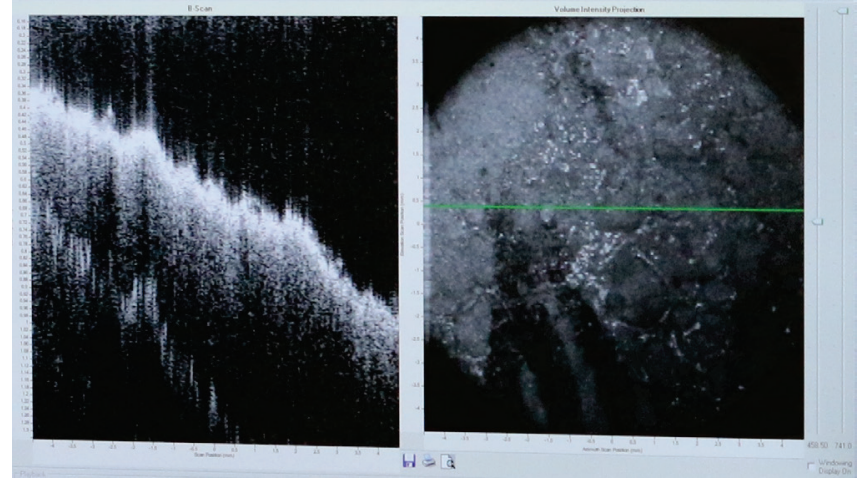

Figure 7. Evisu OCT images showing depth features (left) and spatial area 
The Wasach system is an experimental scanning unit with modular components that is driven on an x:ytable to allow scanning of a larger area. These scans can then be processed and manipulated to allow visualization of a 3D cube (Fig. 8).

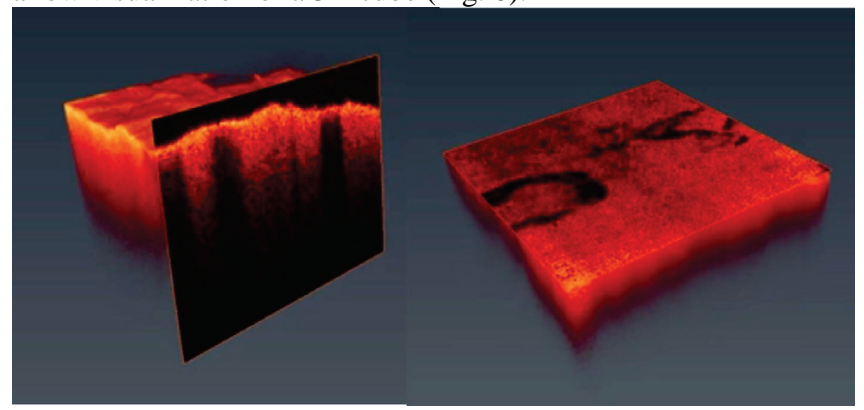

Figure 8. Wasash OCT images showing depth features (left) and spatial area

Amidst ongoing retina and biomedical research, the Duke team processed the images to provide depth and 3D visualizations of small areas of cartonnage that could reveal ink layers and uploaded the data for analysis. Full data processing and metadata were limited for the OCT data sets due to available time with the equipment and data amidst required support for higher priority neonatal, oncological and ophthalmic studies. Based on extensive experience working with these images, they were able to identify real features on the cartonnage sample in the OCT images. SDOCT imaging may indicate two layers of parchment, which differed from artifacts of imaging with reflections of the signals from these layers (Fig 9), but these interpretations require further collaboration by papyrologists, scientists and trained medical professionals. With significant knowledgeable interpretations of the results, experienced users showed areas of contrast between what appear to be text strokes on surfaces, demonstrating that a contrast mechanism between inks and papyrus could be observed, albeit with a trained eye.

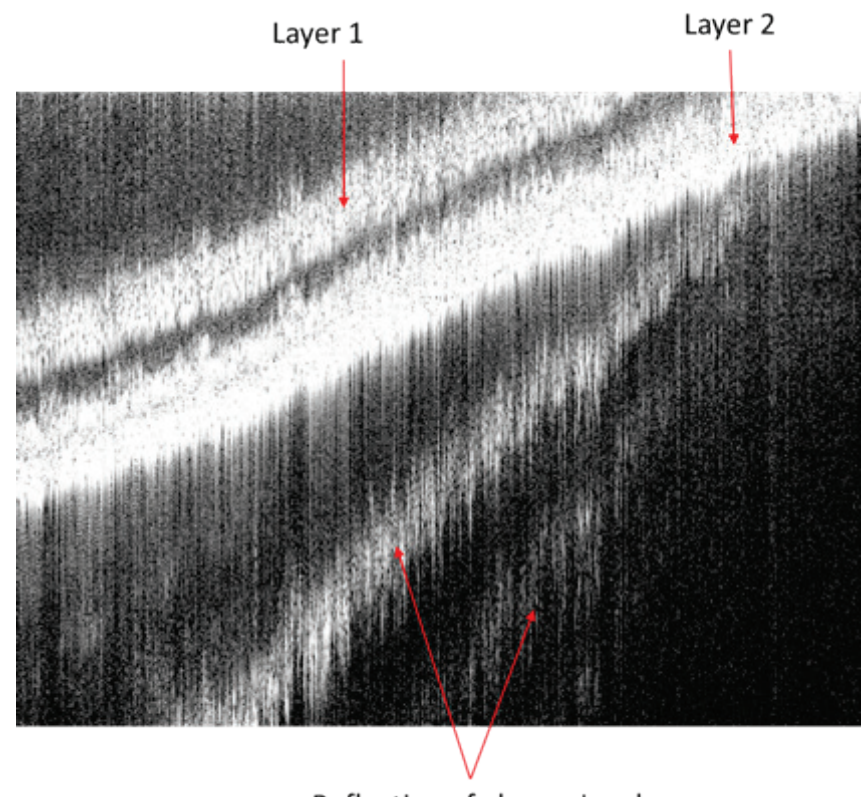

Reflection of above signals

Figure 9. Evisu OCT depth image potentially showing two layers of papyrus

\section{Results}

All the imaging data and metadata was hosted on a public UCL server (https://www.ucl.ac.uk/dh/projects/deepimaging/data) for further review and study, as well as additional research.[5] Due to host system limitations, these data are currently hosted as zip files, but will be hosted in archival form as flat image files with future upgrades. revealed:

Multispectral and OCT imaging of papyri cartonnage

- $\quad$ The internal grain structure of papyri layers in unpigmented regions and areas lacking surface gesso.

- Surface coatings of gesso greatly reduce penetration of light beneath the surface and impeded the ability of spectral imaging to probe the interior of cartonnage.

- $\quad$ Papyrus often has adequate translucency to read text on underlying papyrus layers, but only without diminished translucency from intervening gesso and paint;

- $\quad$ Long wavelengths penetrate best through multiple papyrus layers, but don't excite fluorescence that has potential to be more informative (Fig. 10);

- $\quad$ Fluorescence from cartonnage with gesso was very weak, much weaker than from bare papyrus.

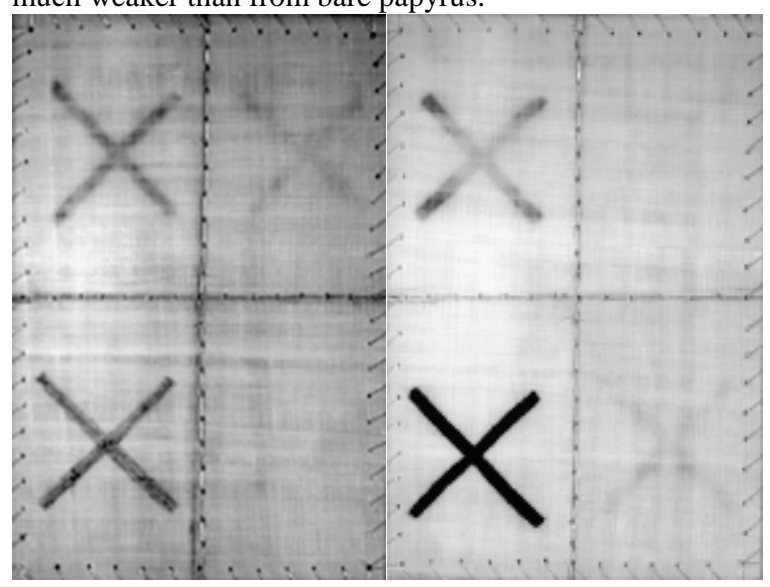

Figure 10. Test samples from UCL, each including crosses at different depths and different inks, imaged in the infrared band, showing penetration through three sheets of papyrus

For papyrus used in this study, translucency was often sufficient to allow detection and visibility of faint surface features (inks or colorants on the facing and reverse sides of the papyrus). Colorants and thicker gesso on the surface could not be penetrated with optical imaging, as they attenuated the light. Papyrus layers alone demonstrate enough translucency to allow detection of internal features or texts, if present. This is particularly true with the longer IR wavelengths, but they do not excite a fluorescent response. Cartonnage with gesso showed fluorescence that was much weaker than that from bare papyrus, and significantly limited short-wavelength penetration. Images captured in the IR wavelength revealed text under only papyrus layers.

The SD-OCT imaging products - image tiles of surfaces regions that require registration to offer larger spatial area - offer sufficient lateral resolution to identify text across a surface. SDOCT imaging showed depth features with parallel bands in crosssectional slices in depth that reveal various features in the cartonnage, including text ink. OCT is unusual in showing both the structure of the papyrus and the presence of the ink 
simultaneously. Because of this, there was more variability in the images from the different sample papyrus. That some spectroscopic features were visible suggests this technique could offer potential to differentiate between inks.

More study will be required to fully assess the ability of OCT to penetrate layers of papyrus, ink and paints and reveal the presence of text. Additional OCT testing conducted by UCL demonstrated that OCT shows both the structure of the papyrus and the presence of the ink. They concluded an optimized OCT system would be required to quantify the ability to differentiate between inks spectroscopically, such as lighter and darker regions on the surface of the top papyrus sheet.[6]

None of the individual imaging techniques alone yield a complete, unambiguous description of the types and depths of features in cartonnage, especially with opaque layers of paint and gesso. Papyrus is somewhat optically translucent to some wavelengths of light through about three layers of papyri, with text enhanced through digital processing. Beyond about three papyri layers the opaque papyri limits penetration of even the longer wavelength IR light.

Given the difficulty of imaging through layers of gesso and paint, this pilot project has clarified that some of the imaging technologies tested could be more usefully applied to book-cover binding, since these artefacts frequently present written papyrus sheets from strata of cartonnage papyri. The lack of a gesso layer offers more potential for optical imaging, including multispectral imaging.

Colorants and thicker gesso on the surface could not be penetrated with multispectral imaging, as they attenuated the light. Papyrus layers alone demonstrate enough translucency to allow detection of internal features or texts, if present. This is particularly true with the longer IR wavelengths, but they do not excite a fluorescent response. Images captured in the IR wavelength revealed text under only papyrus layers. The spectrometer analysis showed that the papyrus is translucent to the longer wavelengths, with some scattering, while the gesso reflects the longer wavelengths. Imaging of cartonnage with gesso showed fluorescence that was much weaker than that from bare papyrus, and significantly limited short-wavelength penetration.

An integrated software tool facilitated visualization of captured and processed multispectral image sequences. Multispectral image processing was conducted with the ImageJbased Paleo Toolbox software provided by Equipoise Imaging to combine, integrate and exploit the captured spectral images, utilizing techniques developed during other cultural heritage studies (Fig. 11). This simple toolbar interface utilizes core ImageJ operations for processing spectral imaging sequences.

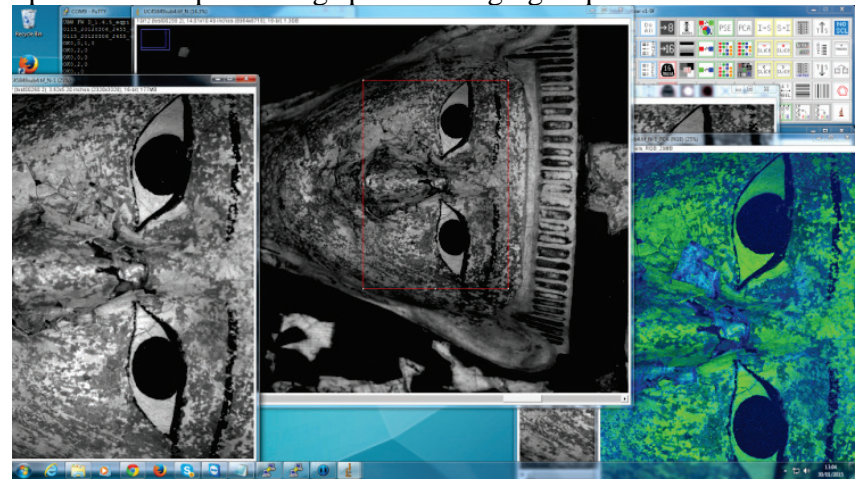

Figure 11. Image processing of mummy mask with integrated software tool

\section{Conclusions}

The various teams from multiple institutions and industry pioneered new methodologies for non-destructive imaging and analysis, sharing of standardized data for interdisciplinary studies, and methods to avoid the destruction of these important cultural heritage objects for textual analysis.

For papyrus used in this study, translucency was often sufficient to allow detection and visibility of faint surface features with each technique, including optically and with additional energy levels. This included detection of inks or colorants on the facing and reverse sides of a layer. While ink was detected under layers of papyrus alone, the leading challenge faced in meeting the program goals was detecting ink text in the layers of papyrus with gesso or paint on the surface. Integrating data from diverse technical imaging systems and work processes proved to be another challenge in working across the different technologies, disciplines and institutions.

Because the optical imaging data of this study indicate that the translucency of gesso is lower than papyrus, the strong reflectance of gesso is problematic for studies of the any internal text and the composition of mummy mask materials. High reflectance with low penetration of light results in data that is strongly dominated by the reflected light. The reflected light and the larger component of noise that accompanies it lowers the signal-to-noise ratio of light scattered from the interior of a material. On the other hand, the correspondingly lower reflectance of gesso at shorter (i.e. visible) wavelengths would present less of a problem in relation to internal scattering by inks that by design have stronger absorbance at shorter (visible) wavelengths. Shorter wavelengths therefor are probably more valuable for detecting strong absorbers (inks) within the mask material, as this higher absorbance provides a contrast mechanism for light that penetrates the bulk material and is reflected or scattered from the papyrus.

Each technique demonstrated potential opportunities for detecting text within cartonnage, but the greatest potential may be the combination of various technologies to tap the strengths of each in an integrated product. Three-dimensional images require more computational power to integrate.

The integration of multispectral data with images from OCT and other imaging methods offered potential to use optical methods to detect inks that are not detectable with any one source alone. This requires the capture of standardized data for registration and post-processing. While ink was detected under layers of papyrus, the leading challenge faced in meeting these goals was detecting ink text in the layers of papyrus with gesso on the surface. The different technologies, disciplines and institutions required integrating data from diverse technical imaging systems and work processes.

More study will be required to fully assess the ability to penetrate more than two layers of papyri, ink and paints and reveal the presence of text. Interpreting SD-OCT features requires significant expertise with the tool to yield information about how to interpret the images and then apply them to gaining information about text within mummy cartonnage. This will require equipment and personnel dedicated to cultural heritage studies, since the preservation of life and vision take precedence for resources in the medical and biomedical engineering environments.

One of the challenges faced in this project was the integration of data and work processes from a variety of scientific tools, disciplines and institutions. This required storage, dissemination, and searchable access to data from instruments that provide output in different formats, some of which were unique to the research 
methods and disciplines. These ranged from basic TIFF images to raw scientific and medical data. While common standards and processes across institutions were encouraged, integrating data from different technologies proved challenging. In addition, many contributors to this project volunteered their time and equipment for imaging and basic processing, but had limited time to spare from their day-to-day responsibilities - ranging from medical personnel preventing blindness to physicists studying elemental changes in bone formation.

Data storage and access to and sharing of timely, complete, and relevant data proved to be a key challenge. This required common standards in a shared technical architecture - which was beyond the capabilities of this quick response program.

Application of these methods to book binding and so-called industrial cartonnage made of layers of papyri and other materials offers more potential than imaging cartonnage with gesso and paint. These are no less in danger than mummy cartonnage, as attested by the fact that the recently published new Sappho fragments probably come from that type of cartonnage, dismounted by the private owner. Dismounting book binding in order to retrieve manuscripts has been a common practice not only of dealers but also of institutional collections, and has led to the destruction of important sources for the history of book-making in the ancient world. While institutions are now proceeding with great care in the eventual dismounting of these artefacts, dealers and collectors' behaviors remain beyond control.

\section{Acknowledgments}

The Deep Imaging Mummy Cases: Non-Destructive Analysis of Multi-Layered Papyrus Project was supported by Arcadia, a charitable fund of Lisbet Rausing and Peter Baldwin. Arcadia serves humanity by preserving endangered cultural heritage and ecosystems and promoting open access. Roberta Mazza at the University of Manchester provided the impetus for this project with her diligent attention and reporting on the destruction of mummy cartonnage by modern collectors.

With Arcadia seed funding, a diverse global team volunteered time and equipment for this project from the fields of digital humanities, medicine, biomedical engineering, particle physics, imaging science, physics, conservation, data management, Egyptology and systems integration. Optical multispectral imaging and spectroscopy was conducted with the support of UCL, Duke University, the University of Manchester, the University of California at Berkeley, the Library of Congress and Equipoise Imaging LLC. The Duke University Papyrology, Digitization, Biomedical Engineering, Medical and Conservation personnel provided additional support for the Duke imaging, including OCT imaging in the Duke Eye Center. Synchrotron Rapid-Scanning Xray Fluorescence imaging was conducted at the SLAC Stanford Synchrotron Radiation Lightsource, x-ray micro Computer Tomography at the Berkeley Advanced Light Source, and Terahertz Imaging at the University of Western Australia.

Authors Michael B. Toth and Cynthia A. Toth are siblings.

Principal Investigator for the project was Melissa Terras.

\section{References}

[1] R. Mazza "Another Indiana Jones? Josh McDowell, mummy cartonnage and biblical papyri.” Faces and Voices. 2014. https://facesandvoices.wordpress.com/2014/05/05/another-indianajones-josh-mcdowell-mummy-cartonnage-and-biblical-papyri/. Accessed 27 Feb 2018.
[2] D. Emery, F.G. France, M.B. Toth, "Management of Spectral Imaging Archives for Scientific Preservation Studies”, Archiving 2009, IS\&T, May 4-7, 137-141 (2009)

[3] F.A. Folgar, E.L. Yuan, S. Farsiu, C.A. Toth, "Lateral and axial measurement differences between spectral-domain optical coherence tomography systems”, Journal of biomedical optics, Vol.19(1), pp.16014 (2014).

[4] H. Hebert, "Multispectral Imaging: What's it Good For?” Duke University Libraries, Bitstreams, April 24, 2017

[5] UCL "Deep Imaging Mummy Cases, The Data” https://www.ucl.ac.uk/dh/projects/deepimaging/data, Accessed 27 Feb 2018.

[6] A. Gibson, et. al., "An assessment of multimodal imaging of subsurface text in mummy cartonnage using surrogate papyrus phantoms”, Heritage Science 2018, 6:7, Feb. 26 (2018)

\section{Author Biography}

M. B. Toth, Program Manager and Systems Integrator; Honorary Research Associate UCL; President, R.B. Toth Associates LLC Cynthia A. Toth, M.D., Joseph A.C. Wadsworth Professor of Ophthalmology, Duke University Eye Center; Professor, Duke University

Pratt School of Engineering, Dept. of Biomedical Engineering William A. Christens-Barry, Engineer, Imaging Scientist, Honorary Research Associate UCL; Principal of Equipoise Imaging LLC Sina Farsiu, Associate Professor, Duke University Department of Biomedical Engineering; Director of the Vision and Image Processing Laboratory Guorong Li, M.D., Research Scientist, Duke University Eye Center Adam Gibson, Professor of Medical Physics, UCL Department of Medical Physics and Biomedical Engineering

Melissa Terras, Principal Investigator; through Sept 2017: Director, UCL Centre for Digital Humanities, Professor of Digital Humanities 